\title{
VERSITA
}

Journal of Official Statistics, Vol. 29, No. 2, 2013, pp. 249-259, DOI: 10.2478/jos-2013-0021

\section{Do Different Listers Make the Same Housing Unit Frame? Variability in Housing Unit Listing}

\author{
Stephanie Eckman ${ }^{1}$
}

\begin{abstract}
Housing unit listing is often used in countries that do not have household or person registries to create frames for household surveys. While several studies have reported the kinds of units and areas that are at risk of overcoverage and undercoverage in such frames, none has looked at variability in the listing process. This article explores this variability by comparing two frames created by trained field staff using the same methods and materials. The overall overlap rate between the two listings is $80 \%$. In nearly all blocks, the listers created different frames, and in more than ten percent of the blocks, the two frames did not overlap at all. In this observational study, the overlap between the two frames is particularly low in the blocks listed using the traditional (from scratch) listing method. There is also evidence that sometimes one lister visited the wrong block. The results show that the listing process can introduce variance into survey data.
\end{abstract}

Key words: Listing; coverage error; coverage variance.

\section{Introduction}

The Total Survey Error framework for survey data summarizes the ways in which a survey estimate, for example a mean, might deviate from the true mean in the population. An estimate may suffer from measurement error if respondents do not answer the question accurately, or from nonresponse error if nonrespondents are different than respondents. An estimate might also suffer from coverage error if the frame from which the sample was selected does not match the population on the measured characteristic. Each error component can introduce bias and/or variance. If we imagine repeating the entire survey multiple times, some errors will always be the same across repetitions (biases) and some will differ (variances) (Andersen et al. 1979; Groves 1989; Lessler and Kalsbeek 1992; Biemer and Lyberg 2003). Measurement error literature has long focused on variances: see, for example, Fellegi (1964); O’Muircheartaigh and Marckward (1980); Schnell and Kreuter (2005). Nonresponse literature has recently begun to consider how the respondent sample changes across repetitions of the recruitment process (O'Muircheartaigh and

\footnotetext{
1 Institute for Employment Research (IAB), Weddingenstraße 20-22 104, 90478 Nuremberg, Germany. Email: Stephanie.Eckman@iab.de

Acknowledgments: This article is based on a chapter of the author's dissertation, written at the Joint Program in Survey Methodology at the University of Maryland. She appreciates the assistance of her committee: Frauke Kreuter (chair), Katharine G. Abraham, J. Michael Brick, and Colm O'Muircheartaigh. The research for the chapter in question was supported by the Census Bureau Dissertation Fellowship, and Tommy Wright, Xijian Liu, Clifford Loudermilk and Aliza Kwiat of the Census Bureau were particularly helpful. Many thanks also to Brady West, Timothy Kennel, Jennifer Sinibaldi, Roger Tourangeau and four reviewers for comments. Any views expressed on statistical, methodological, or operational issues are the author's and not necessarily those of the U.S. Census Bureau.
} 
Campanelli 1999; West and Olson 2010). This article takes a similar approach to the study of coverage error, exploring how listed housing unit frames vary over repetitions of the listing process.

In countries where no register of persons or households is available, listers often create housing unit frames by traveling around the areas selected for the survey and recording the address of every housing unit that they see. In North America, the National Survey of Drug Use and Health (Morton et al. 2006), the General Social Survey (Harter et al. 2010), the National Survey of Family Growth (Lepkowski et al. 2010), the National Children's Survey (Montaquila et al. 2010), the Canadian Labour Force Survey (Statistics Canada 2008), and the Current Population Survey (U.S. Census Bureau 2006) all use listing. Several countries participating in the European Social Survey do so as well (Jowell and the Central Co-ordinating Team 2003, 2005, 2007; Central Co-ordinating Team 2010).

With listed frames, survey researchers worry both about undercoverage, the exclusion of proper housing units from the frame, and about overcoverage, the inclusion of units that do not exist, are not in the selected area, or are not residential. Research has shown that units not occupied at the time of listing and those in small multi-unit buildings (two to nine units) are both undercovered and overcovered (Childers 1992, 1993; Barrett et al. 2002, 2003). Mobile homes are undercovered in listed frames (U.S. Census Bureau 1993; Childers 1993). Undercoverage is also more likely in low-income areas (Manheimer and Hyman 1949; O’Muircheartaigh et al. 2007) and rural areas (O'Muircheartaigh et al. 2007). In addition, households occupied by non-Hispanic black householders have a lower coverage rate than those with non-Hispanic white and other race householders (Barrett et al. 2003). No studies have explored how listed frames vary when the listing task is repeated. Although Kwiat (2009) explores the different actions two listers take when updating an existing frame, that is, whether they confirm, delete or add the same units, these studies do not explicitly compare the frames created by the two listers and do not include blocks where there was no existing frame to update.

Faced with challenging situations, listers may make different judgments and thus produce different frames. For example, in debriefings, six professional listers (none of whom collected the data used in this article) revealed that they use various techniques to count the number of residential units in buildings: some count the mailboxes, others the gas or electricity meters, others the doorbells (Eckman 2010, Appendix F). This article uses data from a repeated listing to explore how similar two frames created by two different listers are. The analyses here do not assert that either frame is more accurate, but instead investigate the overlap between them. The article also investigates the blocks where the two frames do not overlap at all.

\section{Data}

The data for this study come from two listings of a sample of areas carried out by the U.S. Census Bureau in 2007. Two different trained and experienced Census Bureau field representatives listed the housing units in each of these areas. The analyses in this article compare the overlap, or intersection, of the frames made by these listers.

The Census Bureau maintains a Master Address File (MAF), which aims to be a database of all housing units in the United States and serves as the sampling frame for the 
American Community Survey (U.S. Census Bureau 2009). In 2007, the Census Bureau conducted a national evaluation of the MAF's coverage (see Loudermilk and Li 2009 for a discussion of the results). As an add-on, some of the blocks involved in that evaluation were selected for a second listing, carried out by a different lister using the same methods and materials. (Blocks are the smallest geographic units defined by the Census Bureau: they are bounded on all sides by streets, water, railroads or political boundaries.) The subsample gave higher probabilities of selection to blocks with a high rate of growth in the number of addresses available from the U.S. Postal Service, a major component of the MAF. Blocks with no growth were excluded from selection.

Although 301 blocks were selected, only 215 were listed a second time and were found to contain housing units in at least one of the listings (Kwiat 2009). Only these 215 blocks are analyzed in this article, and thus the sample used below is not nationally representative. Unfortunately, none of the housing units listed in this study was selected for a survey, and no data on occupancy status or the characteristics of the residents are available. Also unavailable are any data about which listers were assigned to which blocks, or how many different listers participated in the study.

The listing method used in each block depended on the number of addresses already on the MAF. When the MAF contained addresses for a selected block, the listers were provided with these addresses and updated the list in the field. They added units which lay inside the block but were missing from the MAF, and deleted those that were outside the block or were not housing units. Listers could also move units from one block to another, or simply verify that the unit was correct. This method of listing is called dependent or update listing. When the MAF contained no addresses for a block, listers traveled around the block and created a frame of housing units: this method is called traditional or scratch listing. Fourteen of the blocks in this study were listed using traditional listing because the MAF contained no housing units, and 201 were listed using dependent listing.

In each of the blocks, the two listers used the same listing methods and materials. That is, when the first lister in a given block used traditional listing, so did the second. When the first lister used dependent listing, the second lister did so as well, and the input to the second listing was identical to the input to the first: The second listing was not dependent on the first. The listing software provided listers with a map of the blocks they were to list and displayed the addresses already on the MAF, if any. Assignment of listers to blocks was not random but was driven by location and availability. The second listing was always completed within five months of the first.

The 215 double-listed blocks are located in 44 states and 147 counties and thus not tightly clustered geographically. However, there is one group of 22 contiguous blocks in a large west-coast city that was selected into the sample. All but one of these blocks contained no housing units in the 2000 Census, and they were combined into one cluster prior to selection to ensure that the group as a whole would contain some dwellings. However, these blocks grew substantially during the U.S. housing boom of the last decade, and an average of 97 unique units were listed in each of these blocks (range from one to 510). These 22 high-growth blocks were particularly troublesome for the listers, as discussed below.

Across the blocks, the first frame contained 59,365 housing units and the second contained 60,945 housing units. (In each block, the first frame is simply the one that was 
completed and returned to the central office first.) These counts do not include housing units already on the MAF that were removed by both listers, because these cases do not appear on the final frames. The first step in preparing the data for analysis was matching the two frames. Details on the matching procedures are given in the Appendix.

The two characteristics available for each listed housing unit were whether the unit was a mobile home and whether it was part of a multi-unit structure. Matching revealed some discrepancies in these variables, which required reconciliation. When adding or verifying a unit, a lister can indicate that it is a mobile home. The design of the Census Bureau listing software makes it more likely that listers will fail to flag a mobile home (a false negative) than falsely flag a non-mobile home (a false positive). For this reason, a matched unit was coded as a mobile home if either lister indicated it was. Only $4.3 \%$ of the listed housing units in this study were mobile homes, which is lower than the nationwide occurrence rate of 6.6\% (U.S. Census Bureau; American Community Survey 2008).

Units with any text in the apartment field of the address (except those flagged as mobile homes) were designated as in multi-unit buildings. In one percent of the matched cases, the two listers disagreed about whether a unit should have an apartment designator; these units were marked as multi-units. In this dataset, 58.4\% of the housing units were flagged as in multi-unit buildings, higher than the corresponding nationwide rate of $32.4 \%$ (U.S. Census Bureau; American Community Survey 2008), which points to the over-representation of urban high-growth areas in this study. For those units identified as in multi-unit buildings, information from both listings was used to determine whether the building was small (two to nine units) or large (ten or more units).

In addition, each housing unit on the final two frames can be flagged as either originally on the MAF, or as added by the lister(s). All units in the 14 traditionally listed blocks were by definition added units. In the dependently listed blocks, it was not always straightforward to determine which units were originally on the MAF, because listers sometimes delete a unit that is already on the initial frame and later add that same unit back. In matched cases where one unit was confirmed and the other was added, the unit was not marked as an added unit in the dataset. Overall, $10.2 \%$ of the units were flagged as added in the dataset.

\section{Methods}

The results and discussion below center around the overlap rate, defined as the ratio of the number of housing units in both listings to the number of unique housing units in either listing. Put another way, the overlap rate is the size of the intersection of the two frames divided by the size of the union. (Due to the exclusion of blocks where the two listers both listed no units, the denominator is always non-zero.) An overlap rate of $100 \%$ indicates that the two frames are identical. A rate of $0 \%$ indicates that they have no housing units in common. Thus a high overlap rate means low variability in the listing task and vice versa. (Note that standard measures of inter-rater reliability, such as kappa statistics (Cohen 1969), are not appropriate here, because the data set does not contain housing units in the fourth cell of the two-by-two table, those that neither lister included on the frame.) Due to the nonrepresentative nature of the sample, no significance tests are performed on the overlap rates. 


\section{Results}

The overall overlap rate across all housing units and blocks is $79.9 \%$. That is, more than $20 \%$ of all units listed in this study were included by only one of the two listers. It is clear that the two listers did in fact create different frames, and thus that there is variability in the listing process across replications. This finding is new in the literature.

Table 1 breaks this overlap rate down by housing unit and block characteristics. The overlap rate is higher for units already on the MAF $(81.9 \%)$ than for those that were added $(62.4 \%)$. There are several possible explanations for this finding. Because the added units were harder to match, as explained in the Appendix, matching errors may explain some of the lower overlap rate for the added units, though the careful matching procedures were designed to minimize such errors. The difference could also be related to failure-to-add confirmation bias. Eckman and Kreuter (2011) found that units not on the initial frame were 14.5 percentage points less likely to appear on the frame than those already included. This substantial reduction in listing propensity could explain why one lister added a unit and another did not, leading to a lower overlap rate for the added units.

The blocks in this study contained few mobile homes, and the overlap rate for these units $(73.0 \%)$ is only slightly lower than for the non-mobile home cases $(80.2 \%)$. The difference between the overlap rates for single-family $(83.5 \%)$ and multi-family units $(77.3 \%)$ is also small. However, breaking the multi-unit buildings into small and large shows that the overlap rate for units in small buildings is quite a bit lower $(66.0 \%)$. These results are consistent with previous research that finds small multi-unit buildings to be difficult to list correctly (Childers 1993), as well as with the listers' own statements in the debriefings.

The overlap rate in this study does not differ between rural and urban blocks, despite previous findings that rural areas tend to be undercovered. This finding may mean that all listers are affected similarly by the challenges of rural listing, undercovering the same units. However, the sample used in this study itself underrepresents rural areas, and thus this result may be misleading.

Table 1. Overlap rates, by housing unit and block characteristics

\begin{tabular}{lcr}
\hline & Overlap rate & \multicolumn{1}{c}{$n$} \\
\hline On input list & $81.9 \%$ & 60,042 \\
Added & $62.4 \%$ & 6,838 \\
Mobile home & $73.0 \%$ & 2,868 \\
Non-mobile home & $80.2 \%$ & 64,012 \\
Single family & $83.5 \%$ & 27,825 \\
Multi-Unit & $77.3 \%$ & 39,055 \\
$\quad$ Small, 9 or fewer units & $66.0 \%$ & 4,087 \\
$\quad$ Large, more than 9 units & $78.7 \%$ & 34,968 \\
Rural block & \\
Urban block & $79.8 \%$ & 12,802 \\
Traditional listing & $79.9 \%$ & 54,078 \\
Dependent listing & $13.6 \%$ & 523 \\
a Census 2000 Summary File data & $80.4 \%$ & 66,357 \\
\hline
\end{tabular}


The 14 blocks listed via traditional listing have much lower overlap rates than the blocks listed with dependent listing (13.6\% vs. 80.4\%). However, listing method was not randomly assigned in this study and this result should be interpreted with caution. It could be other block attributes, rather than the listing method itself, which drive the low overlap rates.

The overlap rates do vary quite a bit across the 215 blocks in the study (Figure 1). There are only 20 blocks where the two frames overlap completely, in the upper right corner, and these are small blocks: None has more than 60 unique housing units, and 15 have ten or fewer units. In 116 blocks, the overlap rates are $80 \%$ or higher. However, these tend to be the largest blocks, and they represent $74 \%$ of the unique housing units in the dataset.

There are 25 blocks in the lower left corner of Figure 1 where the two listers created frames that do not overlap at all. In most (22) of these blocks, the overlap rate is $0 \%$ because one lister listed no units. In other words, one lister found no housing units in the assigned block, while the other found one or more. It is possible that both listers saw the structures, but one thought they were nonresidential and thus did not include them. Another possible explanation for the $0 \%$ overlap is that one lister was in the wrong block. Inspection of the pattern of housing unit numbers and street names against Google Maps strongly suggests that in at least ten of the 22 blocks, one lister included units outside the selected block. That is, in nearly $5 \%$ of the blocks listed in this study, one lister seems to have created a frame for the wrong block. When listers systematically list the wrong block, they overcover the housing units in the wrong block, giving them more than one chance of selection, and undercover the units in the right block, giving them no chance of selection.

The blocks listed via traditional listing are overrepresented among the $0 \%$ overlap blocks. Ten of the 25 blocks with $0 \%$ overlap rates were traditionally listed, and these ten are the majority of the 14 traditionally listed blocks in the study, which explains the low overlap rate for traditional listing in Table 1. Furthermore, of the ten blocks where evidence suggests that one lister was in the wrong area, eight were listed via traditional listing. The number of blocks and units listed via traditional listing is small in this study,

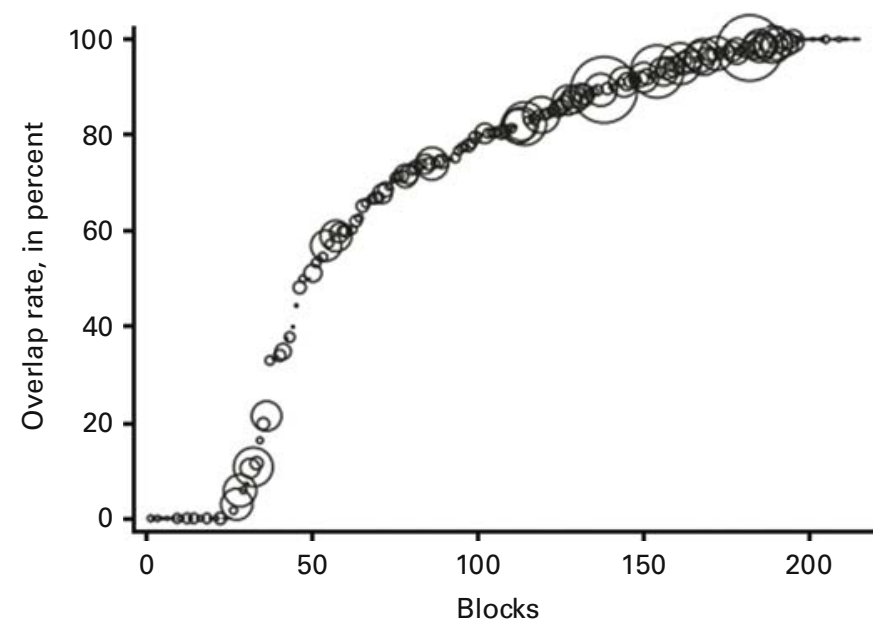

Fig. 1. Block-level overlap rates; horizontal axis is the 215 double-listed blocks, sorted by overlap rate; size of point is relative to the count of unique housing units 
due to the nonrepresentative sample. Nevertheless, these results point to high variability in the traditional listing process.

In the debriefings, the listers provided a possible explanation for the connection between traditional listing and the wrong block problem. Several said that they find it easier to locate the selected block when using dependent listing: They simply look for the housing unit listed first on the existing frame and list the block it is in (Eckman 2010, Appendix F). When doing traditional listing, listers cannot use this technique, which may increase the likelihood that they list the wrong block, and thus explain the low overlap rate for traditional listing found in this study.

The 22 contiguous West Coast blocks are also overrepresented among the $0 \%$ overlap blocks. The overlap rate across these blocks is only $44.9 \%$, and in thirteen of these blocks the two frames had no units in common. The housing unit stock in these blocks increased by more than $200 \%$ from the 2000 to 2010 census, indicating a good deal of growth at some point in the decade, and raising the possibility that real change in the field occurred between the first and second listings, which were at most five months apart. However, close inspection of the frames in these blocks in conjunction with online mapping resources revealed that one lister was confused about the block boundaries. An interstate highway runs through this neighborhood and cuts out very narrow strips of land between the highway and the frontage road on both sides. It appears that one lister did not recognize these strips as blocks and thus was one block off when listing portions of the area.

\section{Discussion and Conclusion}

This repeated listing study finds that different listers do produce different housing unit frames. Listers disagreed about the inclusion of added units in the frame more than those already on the MAF, and about those in small multi-unit buildings more than single family homes. There was also substantial variation in the overlap rates across the 215 blocks. Traditional listing was more likely to be associated with low overlap than dependent listing, and there is some evidence that listers using traditional listing were more likely to list the wrong block. Because the sample for this study is not nationally representative and the data are observational, the findings may not generalize broadly, but substantial variability in the housing unit listing process is a result not seen before in the literature, and should be of interest to all studies using listing.

The most troubling finding to come from this investigation is that listers may at times be in different blocks, particularly when doing traditional listing. If this finding is replicated in larger studies, several procedural changes could help prevent and detect such errors. First, lister training should include material and job aids on locating the assigned block based on the provided map. Second, quality control procedures should be revised to detect when the wrong block is listed. The Census Bureau's listing check procedure, which sends a senior field representative back to relist the blocks, could catch these mistakes if the second lister does not herself use the addresses listed by the first to locate the block to be checked. The National Survey of Family Growth (NSFG) reviews all of its listings by comparing the street names and numbers against external sources such as Google Maps. This sort of review may be more likely to catch the wrong-block errors, and is less costly than in-field relisting. Third, survey researchers may wish to avoid traditional listing when 
possible. Less traditional listing may mean fewer errors of listers misreading their maps and listing the wrong block. However, there are two important caveats to this recommendation. First, the addresses on the initial frame may themselves be in the wrong block, due, for example, to geocoding error (Eckman and English 2012). Second, dependent listing has its own vulnerability, namely confirmation bias (Eckman and Kreuter 2011).

This unique double listing dataset has demonstrated that replications of the housing unit listing process do result in different frames. If the residents of the housing units included by the first lister are different, in ways that are captured in the survey items, from those included by the second lister, then the variability in the frames would introduce coverage variance. This study was not able to estimate coverage variance, due to the lack of survey data for all of the listed units, but future studies should aim to do so. Future research should also experimentally manipulate the listing method to better understand how the two methods work. Taking a cue from research into interviewers' contributions to measurement and nonresponse error, coverage studies should collect information about the listers themselves to explore how their characteristics, such as experience, training, and attitudes, affect housing unit listing.

\section{Appendix}

The quality of the matching procedures is central to the results presented in the article. The 59,365 housing units on the first listings and the 60,945 unit on the second were run through a three step matching process. The guiding principle throughout the process was whether the same housing unit would be interviewed if the two addresses were selected. For example, unit A and unit 1 at the same address most likely refer to the same unit, and selecting either one would lead to the same unit being approached for an interview, thus these two units were matched. None of the matching steps made use of recent advances in statistical or probabilistic matching (Herzog et al. 2007). While these techniques are appropriate for large-scale matching projects, they are not necessary here where the dataset is rather small, especially within each block, and all addresses can be compared visually.

In the blocks where the listers used dependent listing, matching the units on the input list that each lister verified was straightforward. Every housing unit on the MAF has a unique ID, and the first step simply matched units on the two frames by this ID, identifying housing units that both listers verified. These are the majority of all of the matches identified, as shown in Table 2.

All housing units on the MAF that were not matched in step one and all those added by the listers moved onto step two, which consisted of seven matching routines programmed in SAS 9.1. These routines took advantage of the fact that listers parsed addresses into eight fields in the listing software. The first pass required that all of the address fields, plus block number and apartment identifier, match exactly. Subsequent passes dropped fields from the matching criteria. For example, the second pass did not require a match on the direction prefix field, so that 932 E Elm St would match to 932 Elm St. The seventh pass would match 115 Bryant Ave to 115 Bryant St if they were listed in the same block and still unmatched. Identifying more precise matches first ensured that a low quality match 
Table 2. Matches identified, by matching step

\begin{tabular}{lrc}
\hline Matching step & Housing units & $\%$ of all matches \\
\hline Step 1: Units from MAF & 44,753 & $83.8 \%$ \\
Step 2: Address matches, in SAS & 8,428 & $15.8 \%$ \\
Pass 1: block, unit no., \& all address fields & 7,849 & \\
Pass 2: drop direction prefix (N, E) & 12 & \\
Pass 3: also drop extension & 0 & \\
Pass 4: also drop direction (W, NE) & 6 & \\
Pass 5: also drop house no. suffix (A, 1/2) & 0 & $0.5 \%$ \\
Pass 6: also drop street type prefix & 531 & \\
Pass 7: also drop street type (St, Ave, Dr) & 249 & \\
Step 3: Address matches, manual review & 53,430 & \\
\hline Total matched units & 13,450 & \\
Total unmatched units &
\end{tabular}

would not crowd out a better match. All passes required that the block number, house number, street name, and apartment designator match exactly. These matching routines identified 8,428 matches, more than $90 \%$ of those in the first (most precise) pass (see Table 2).

Units still unmatched after Step 2 were output for manual matching. This step caught many spelling and parsing errors as well as different street names (Route 93 versus Main St) and apartment designators (A, B, C versus 1, 2, 3). (In several cases, the spelling and parsing errors in the datasets were fixed and the Step 2 matching routines rerun. The match counts in Table 2 reflect the results after these cleaning steps were applied.) When one lister included two units at an address and the other only one, the single unit was matched to the first unit and the second unit left unmatched. This step identified 249 additional matches (Table 2).

The distance between the geographic coordinates collected by the two listers for each of the matched pairs provides a quality check on the matching. The average distance between the points was 0.06 kilometers, the median was 0.03 , and the maximum was 3.3 kilometers. However, the largest distances occurred among the most precise matches (Step 1). In the less precise steps, the distance between the units was always less than one kilometer. Variability in the geocoding of points (Sando et al. 2005; Listi et al. 2007) may explain some of the large distances between matched points.

\section{References}

Andersen, R., Kasper, J., and Frankel, M.R. (1979). Total Survey Error. San Francisco: Jossey-Bass.

Barrett, D.F., Beaghen, M., Smith, D., and Burcham, J. (2002). Census 2000 Housing Unit Coverage Study. Proceedings of the Section on Survey Research Methods: American Statistical Association, 146-151. 
Barrett, D. F., Beaghen, M., Smith, D., and Burcham J. (2003). Census 2000 Housing Unit Coverage Study. Technical Report Census 2000 Evaluation O.3, U.S. Census Bureau, Washington, D.C.

Biemer, P.P. and Lyberg, L.E. (2003). Introduction to Survey Quality. New York: Wiley-Interscience.

Central Co-ordinating Team (2010). European Social Survey Round 3 2008/2009. Final Activity Report ESS4e03.0, City University London.

Childers, D.R. (1992). The 1990 Housing Unit Coverage Study. Proceedings of the Section on Survey Research Methods: American Statistical Association, 506-511.

Childers, D.R. (1993). Coverage of Housing in the 1990 Decennial Census. Proceedings of the Section on Survey Research Methods: American Statistical Association, 635-640.

Cohen, J. (1969). Weighted Kappa: Nominal Scale Agreement with Provision for Scaled Disagreement or Partial Credit. Psychological Bulletin, 70, 213-220.

Eckman, S. (2010). Errors in Housing Unit Listing and Their Effects on Survey Estimates. Ph. D. thesis, University of Maryland.

Eckman, S. and English, N. (2012). Creating Housing Unit Frames from Address Databases: Geocoding Precision and Net Coverage Rates. Field Methods, 24, 399-408.

Eckman, S. and Kreuter, F. (2011). Confirmation Bias in Housing Unit Listing. Public Opinion Quarterly, 75, 139-150.

Fellegi, I.P. (1964). Response Variance and Its Estimation. Journal of the American Statistical Association, 59, 1016-1041.

Groves, R.M. (1989). Survey Errors and Survey Costs. Hoboken, N.J: John Wiley and Sons.

Harter, R., Eckman, S., English, N., and O'Muircheartaigh, C. (2010). Applied Sampling for Large-Scale Multi-Stage Area Probability Designs. Handbook of Survey Research, P.V. Marsden and J. Wright (eds). (Second ed). Bingley: Emerald, 169-197.

Herzog, T.N., Scheuren, F.J., and Winkler, W.E. (2007). Data Quality and Record Linkage Techniques. New York: Springer.

Jowell, R. and the Central Co-ordinating Team (2003). European Social Survey 2002/2003. Technical Report ESS1e06.1, London City University.

Jowell, R. and the Central Co-ordinating Team (2005). European Social Survey Round 2 2004/2005. Technical Report ESS2e03.1, London City University.

Jowell, R. and the Central Co-ordinating Team (2007). European Social Survey Round 3 2006/2007. Technical Report ESS3e03.2, London City University.

Kwiat, A. (2009). Examining Blocks with Lister Error in Area Listing. Proceedings of the Section on Survey Research Methods: American Statistical Association, 2546-2557.

Lepkowski, J.M., Mosher, W.D., Davis, K., Groves, R.M., and Hoewyk, J.V. (2010). The 2006- 2010 National Survey of Family Growth: Sample Design and Analysis of a Continuous Survey. Vital Health Statistics, 2(150).

Lessler, J.T. and Kalsbeek, W.D. (1992). Nonsampling Error in Surveys. Hoboken, N.J.: John Wiley and Sons.

Listi, G.A., Manhein, M.H., and Leitner, M. (2007). Use of the Global Positioning System in the Field Recovery of Scattered Human Remains. Journal of Forensic Science, 52, 11. 
Loudermilk, C.L. and Li, M. (2009). A National Evaluation of Coverage for a Sampling Frame Based on the Master Address File (MAF). Proceedings of the Section on Survey Research Methods: American Statistical Association, 1721-1734.

Manheimer, D. and Hyman, H. (1949). Interviewer Performance in Area Sampling. Public Opinion Quarterly, 13, 83-92.

Montaquila, J.M., Brick, J.M., and Curtin, L.R. (2010). Statistical and Practical Issues in the Design of a National Probability Sample of Births for the Vanguard Study of the National Children's Study. Statistics in Medicine, 29, 1368-1376.

Morton, K.B., Hunter, S.R., Chromy, J.R., and Martin, P.C. (2006). Population Coverage in the National Survey of Drug Use and Health. Proceedings of the Section on Survey Research Methods: American Statistical Association, 3441-3446.

O’Muircheartaigh, C. and Campanelli, P. (1999). A Multilevel Exploration of the Role of Interviewers in Survey Non-Response. Journal of the Royal Statistical Society. Series A (Statistics in Society), 437-446.

O’Muircheartaigh, C. and Marckward, A.M. (1980). An Assessment of the Reliability of World Fertility Study Data. Proceedings of the World Fertility Survey Conference, 3, 305-379.

O’Muircheartaigh, C.A., English, E.M., and Eckman, S. (2007). Predicting the Relative Quality of Alternative Sampling Frames. Proceedings of the Section on Survey Research Methods: American Statistical Association, 551-574.

Sando, T., Mussa, R., Sobanjo, J., and Spainhour, L. (2005). Quantification of the Accuracy of Low Priced GPS Receivers for Crash Location. Journal of the Transportation Research Forum, 44, 19-32.

Schnell, R. and Kreuter, F. (2005). Separating Interviewer and Sampling-Point Effects. Journal of Official Statistics, 21, 389-410.

Statistics Canada (2008). Methodology of the Canadian Labour Force Survey. Technical Report 71-526-X, Statistics Canada.

U.S. Census Bureau (1993). Programs to Improve Coverage in the 1990 Census. Technical report. $1990 \mathrm{CPH}-\mathrm{E}-3$.

U.S. Census Bureau (2006). Technical Paper 66: Design and Methodology, Current Population Survey. Technical report.

U.S. Census Bureau (2009). Design and Methodology American Community Survey. Technical report, U.S. Census Bureau, Washington, D.C.

U.S. Census Bureau; American Community Survey (2008). Selected Housing Characteristics: 2008, 1-Year Estimates. Generated by author using American FactFinder < http://factfinder.census.gov > (Accessed 28 December 2010).

West, B. and Olson, K. (2010). How Much of Interviewer Variance is Really Nonresponse Error Variance?, Public Opinion Quarterly, 74, 1027-1045.

Received November 2011

Revised February 2012

Accepted January 2013 